\title{
Change of hydraulic conductivity of cohesive soils due to consolidation treatments with expanding resins
}

\section{Variazioni di conducibilità idraulica in terreni coesivi soggetti a trattamenti di consolidazione con resine espandenti}

Tiziana Apuani, Marco d'Attoli, Marco Masetti, Federico Fischanger

Riassunto: Assestamenti e cedimenti nei terreni sottoposti a carico di fondazione sono spesso dovuti a cause naturali e/o antropogeniche, come consolidamenti differenziali, fenomeni di infiltrazione, periodi di siccità alternati a piogge abbondanti, crescita di radici, vibrazioni indotte dal traffico veicolare, scavi o difetti di costruzione. Tecniche di consolidamento e stabilizzazione mediante iniezione nel terreno di fondazione di resine espandenti in poliuretano sono state utilizzate efficacemente per mitigare o addirittura risolvere questo problema. L'efficienza del trattamento di iniezione di resina è stata ben documentata anche grazie allo sviluppo di una procedura di pianificazione e monitoraggio che affianca ai tradizionali test geotecnici le tomografie di resistività elettrica, estremamente sensibile alla presenza di acqua o vuoti nel terreno. La combinazione delle metodologie di indagine consente di riconoscere l'effetto delle iniezioni in termini di compattazione del suolo e migrazione dell'acqua, fattore chiave per la comprensione del fenomeno e la pianificazione di interventi risolutivi.

Il presente studio rappresenta il seguito di un lavoro precedente finalizzato alla valutazione dell'incremento delle prestazioni geotecniche di terreni coesivi trattati mediante iniezione di resine espandenti (le stesse e nello stesso sito) e lo integra focalizzan-

Keywords: : hydraulic conductivity, cohesive soil, consolidation treatments, expanding resin, soil stabilization.

Parole chiave: conducibilità idraulica, terreno coesivo, trattamento di consolidamento, resine espandenti, stabilizzazione del terreno.

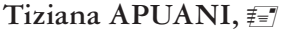

Dipartimento di Scienze della Terra "A. Desio"

Università degli Studi di Milano

tiziana.apuani@unimi.it

\section{Marco D'ATTOLI}

GEOSEC s.r.l. - Parma

ufficiotecnico@geosec.it

\section{Marco MASETTI}

Dipartimento di Scienze della Terra "A. Desio"

Università degli Studi di Milano

marco.masetti@unimi.it

\section{Federico FISCHANGER}

Geostudi Astier S.r.l. - Livorno, Italy

federico@fischanger.it

Ricevuto/Received: 31 July 2018-Accettato/Accepted: 14 September 2018 Pubblicato online/Published online: 27 September 2018

This is an open access article under the CC BY-NC-ND license:

http://creativecommons.org/licenses/by-nc-nd/4.0/

(C) Associazione Acque Sotterranee 2018 do l'attenzione sulla quantificazione gli effetti indotti sulle proprietà idrologiche dei terreni coesivi, attraverso la misura delle variazioni di conducibilità idraulica satura.

Test di permeabilità in celle triassiali, a differenti condizioni di sforzo e gradiente idraulico, sono state eseguite su campioni prelevati in un campo prova sperimentale a scala reale di "terreno naturale" (non trattato, campioni indisturbati) e "terreno trattato" (campioni indisturbati), prima e dopo l'iniezione di resina (MAXIMA® di GEOSEC ${ }^{\circledR}$ ). Il terreno studiato è classificato come argilla con limo molto consistente e apparentemente sovraconsolidato.

Dallo studio emerge come il trattamento sia in grado di modificare le caratteristiche di conducibilità idraulica del terreno. Le resine iniettate seguano in parte piani di debolezza preesistenti ed in parte impregnano in modo capillare masse omogenee e continue di terreno, conferendogli, oltre alla già documentata maggior resistenza meccanica, una minor conducibilità idraulica. Le indagini di resistività elettrica consentono di apprezzare i volumi affetti dal trattamento a cui attribuire le variazioni delle proprietà misurate.

Abstract: Settlements of ground subjected to foundation load are often due to both natural and/or anthropogenic causes, such as differential consolidation, seepage, periods of drought alternating with heavy rainfalls, growth of tree roots, sewer leaks, vibrations induced by vehicle traffic, excavations, or construction defects. Techniques of soil consolidation and stabilization by means of injection of polyurethane expanding resins have been efficiently used to mitigate or even solve this issue. The efficiency of the resin injection treatment has been well documented also thanks to the develop of a procedure of planning and monitoring which combines traditional geotechnical tests with the application of electrical resistivity tomography, which is strongly sensitive to the presence of water or voids.

The combination of these survey methodologies allows to recognize the effect of injection in terms of soil compaction and water migration, a key factor for understanding the phenomenon and planning of resolutive interventions.

The present study follows a previous work aimed at evaluating the increase in the geotechnical performance of cohesive soils treated by injection of expanding resins (same soil, resin, at the same site) and integrates it by focusing on the quantification of the effects of consolidation treatments, by means of expanding resins injection, on hydrologic properties of cohesive soils, focusing on saturated hydraulic conductivity measurements.

Laboratory permeability test, performed in triaxial cell at different stress conditions and hydraulic gradients, were performed 
on undisturbed samples collected on "natural (non-treated)" and "treated soils", before and after the injection of resin (MAXIMA ${ }^{\circledR}$ by GEOSEC $($ ) ) in a full-scale test site characterized by the presence of continuous and homogeneous cohesive soils, (clay and silts). The investigated soil is classified as $\mathrm{CH}$ or CL soil, very firm and apparently overconsolidated.

The study shows how the treatment is able to modify the characteristics of hydraulic conductivity of the soil. The injected resins partly follow pre-existing weaknesses and partly impregnate homogeneous and continuous masses of soil in a capillary way, giving it, in addition to the already documented greater mechanical strength, a lower hydraulic conductivity. The electrical resistivity investigations allow to appreciate the volumes affected by the treatment to which the variations of the measured properties can be attributed.

\section{Introduzione}

Lapplicazione di tecniche di consolidamento/stabilizzazione dei terreni di fondazione con iniezione di resine anche espandenti è notevolmente aumentata negli ultimi anni, accompagnata dallo sviluppo di nuovi materiali e metodi appositamente studiati per questa innovativa applicazione (Occhi et al., 2006; Occhi et al., 2008; Occhi et al., 2017). I primi impieghi risalgono ormai alla seconda metà degli anni 50 negli Stati Uniti (Oertel, 1993) per il riempimento di cavità sotterranee e a seguire negli anni '90 in Europa per il sollevamento di pavimentazioni cedute. Oggi esistono sul mercato diverse tecniche ed applicazioni più evolute e dedicate in particolare ai terreni di fondazione delle costruzioni; tra le procedure più diffuse consideriamo quelle di consolidamento mediante iniezione a diffusione libera di una miscela polimerica bicomponente eco compatibile in grado di espandersi più o meno rapidamente per reazione chimica esotermica nel terreno.

L'interesse suscitato da questo procedimento risiede principalmente nella sua elevata efficienza, anche a lungo termine; nella sua limitata invasività, rapidità di intervento, flessibilità di impiego e possibilità di lavorare in spazi ristretti e confinati; nella garanzia di compatibilità ambientale oltre al fatto che il trattamento vantaggiosamente non appesantisce il terreno ma favorisce la riduzione dei vuoti e del contenuto idrico interstiziale.

Il dissesto del terreno di fondazione trova invece ragione in molte cause naturali e talvolta antropiche. Periodi di siccità alternati a forti precipitazioni, ma anche perdite fognarie, traffico veicolare pesante, scavi vicino alla costruzione, crescita delle radici degli alberi ad alto fusto in prossimità delle fondamenta, fenomeni di suzione e variazione dei carichi applicati, sono solo alcune delle cause che possono dare origine a cedimenti del terreno. L'interazione fra acqua e terreno di fondazione è sicuramente uno dei meccanismi più importanti i cui effetti negativi sono tecnicamente difficili da contrastare e monitorare, soprattutto se è presente una frazione argillosa significativa. I terreni argillosi, infatti, hanno la capacità di assorbire grandi quantità di acqua, con conseguente notevole aumento di volume, mentre al contrario la perdita di acqua si manifesta in una diminuzione del volume del terreno. Quando periodi di pioggia intensa si alternano a periodi prolungati di siccità, il volume sensibile del terreno viene ciclicamente sottoposto a rigonfiamento e ritiro (Vincent, 2009), che porta alla creazione di vuoti e riduzione del volume dell'aggregato terra, in dipendenza dalla natura dei minerali argillosi e della chimica del fluido, quindi al rischio di cedimento differenziale verticale per le costruzioni soprastanti con effetti dannosi alle strutture edificate. Terreni granulari, invece, possiedono una maggiore permeabilità e l'acqua circola liberamente, eventualmente trasportando particelle fini. Questa circolazione, se sufficientemente intensa, può portare progressivamente alla creazione di vuoti e cavità, ed eventualmente al dissesto della fondazione. Naturalmente i cedimenti del terreno possono essere conseguenza anche di problemi strutturali come ad esempio incremento dei carichi per successivi interventi di sopraelevazione, errata progettazione o esecuzione delle opere strutturali. Poiché cambiamenti del contenuto di fluidi e/o vuoti, inducono cedimenti nel sottosuolo, lo scopo principale dell'iniezione di resine anche espandenti è di ristabilire la continuità e la compattazione del suolo intorno e sotto alla fondazione ceduta. In altre parole si tratta di un processo di ri-organizzazione spaziale dei vuoti a cui consegue un miglioramento delle proprietà di resistenza e deformabilità della massa trattata (Apuani et al., 2011; Apuani et al., 2015) oltre che delle condizioni idriche, grazie al riempimento degli stessi, consentendo al terreno ceduto di ripristinare le condizioni iniziali di stabilità (Rittgers et al., 2010; Fischanger et al, 2013). Sebbene l'uso di tecniche di consolidamento a base di resine sia sempre più in crescita, la comprensione completa del processo necessita di ulteriori approfondimenti per meglio comprendere le modalità di azione del trattamento e valutare il comportamento del terreno durante e dopo il trattamento.

Alcuni studi sono stati dedicati ad esplorare le variazioni nelle proprietà geotecniche, idrogeologiche e geofisiche dei terreni, durante e dopo il trattamento, attraverso l'analisi a posteriori in siti di prova appositamente realizzati (Apuani et al. 2011, Apuani et al. 2015) o in casi reali di applicazione (Santarato et al., 2011; Fischanger et al., 2013; Rittgers, et al., 2010). Solo pochi autori hanno applicato un approccio teorico per prevedere l'efficacia del trattamento (Dei Svaldi et al., 2005). Ne risulta che l'argomento necessita di ulteriori approfondimenti.

A causa della complessità del sistema (terreno/resina), è inevitabile che ogni caso di trattamento sia dipendente dal contesto geotecnico, nonché dal prodotto e dalla procedura specifica utilizzata nell'intervento, l'efficacia del quale deve essere monitorata specialmente durante e dopo l'iniezione, attraverso la visualizzazione possibilmente in time lapse tridimensionale del sottosuolo. Esistono numerose tecniche non-invasive, messe a disposizione dalla Geofisica, che possono offrire una visione 3D del sottosuolo investigato ripetuta nel tempo, ad esempio le tecniche sismiche, le tecniche basate sulla resistività elettrica, il georadar. Il consolidamento del 
terreno, attraverso la riduzione dell'acqua in eccesso e/o il riempimento dei vuoti, mediante trattamento con iniezione di resine, si presta particolarmente bene al monitoraggio tramite tecniche geofisiche basate sulla misura ripetuta nel tempo della resistività elettrica, essendo questa proprietà fisica molto sensibile sia alla presenza di vuoti che al contenuto d'acqua presente.

Uno dei punti cruciali che si affronta nella presente pubblicazione è la quantificazione della variazione di conducibilità idraulica indotta dal trattamento con resine in terreni coesivi. Il problema, che nei casi reali di applicazione viene indirettamente monitorato mediante controlli geofisici di tomografia della resistività elettrica, viene in questa sede affrontato eseguendo prove di permeabilità in cella triassiale su campioni di argilla prelevati in un campo prove appositamente realizzato, prima e appena dopo l'esecuzione di un trattamento di iniezione.

\section{Metodologia \\ II campo prove}

Il campo prove è sito in località San Martino Sinzano (Comune di Collecchio - PR) (Fig.1a). Si tratta di un campo piano ad uso agricolo, oggi incolto, nelle cui adiacenze gli autori avevano già condotto uno studio finalizzato alla valutazione delle prestazioni tecniche dei terreni consolidati con le stesse resine usate nel presente studio (Apuani et al, 2011; Apuani et al., 2015) e a cui nel seguito si farà spesso riferimento poiché integra con dati geotecnici i risultati presentati in questo scritto. In particolare in quello studio (nel seguito spesso citato come "studio precedente"), trincee esplorative, prove penetrometriche CPTU, indagini di tomografia elettrica verticale, campionamenti e prove geotecniche su campioni indisturbati, hanno consentito di ricostruire i caratteri stratigrafici fino alla profondità di $3 \mathrm{~m}$ e di riconoscerne l'omogeneità laterale, caratteri tutti confermati dalle indagini presentate in questa nota.

Larea di studio si trova a sud-ovest di Parma, nel comune di Collecchio, in posizione di avanfossa rispetto alla catena appenninica che si innalza immediatamente a sud della suddetta località e si sviluppa in direzione NWSE cingendo così la parte meridionale della Pianura Padana. Il sottosuolo è contraddistinto da un potente accumulo di sedimenti alluvionali quaternari che appoggiano in discordanza semplice sul substrato di sedimenti marini del Pliocene Superiore e del Pleistocene Inferiore, direttamente coinvolti nel presente studio (Fig. 1b). Lo spessore di questi sedimenti si assottiglia marcatamente procedendo verso SW e raggiunge il suo spessore minimo in prossimità dell'abitato di Collecchio. In superficie la maggior parte del territorio è ricoperto da paleosuoli a diverso grado di evoluzione. Si tratta prevalentemente di limi e argille con locale presenza di ghiaie e sabbie che mediamente raggiungono la profondità di 3 metri, su conglomerati e ghiaie appartenenti alle unità alluvionali quaternarie.

Il campo prove è stato delimitato isolando due porzioni attigue: la prima riservata alle indagini in condizioni naturali, la seconda al terreno interessato da iniezione di resina.

\section{Indagini e campionamenti}

La sperimentazione si è realizzata in 4 fasi: i) indagini pre trattamento, ii) iniezioni con resine espandenti, iii) indagini post intervento, iv) prove di laboratorio.

In previsione di eseguire un'unica iniezione a $1.10 \mathrm{~cm}$ dal p.c., le indagini si concentrano nell'orizzonte di terreno fino a $-3 \mathrm{~m}$ di profondità. L'estensione dell'area di influenza del trattamento dipende da diversi fattori, tra cui natura e condizioni fisiche del terreno, (porosità, compattazione ...), quantitativi e pressioni di iniezione delle resine. L'esperienza

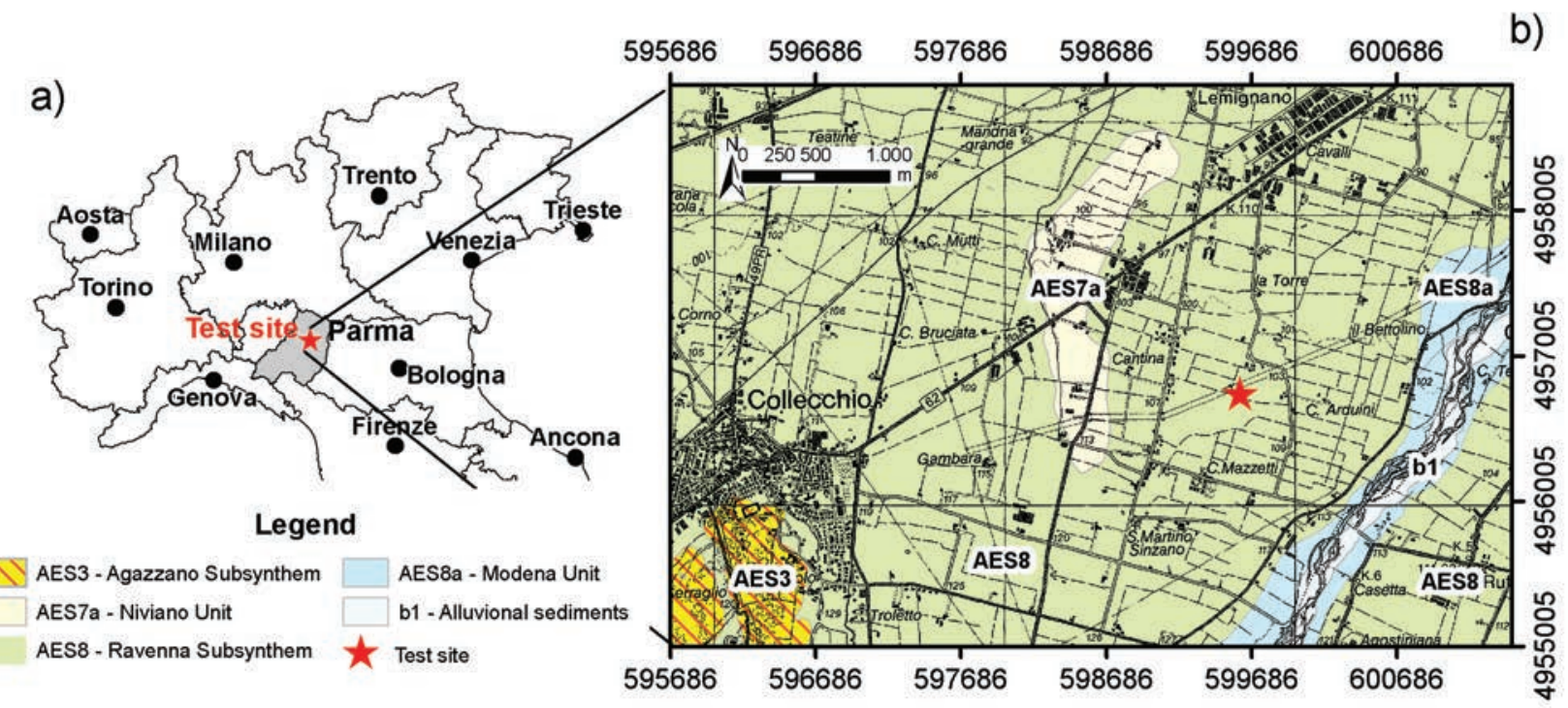

Fig. 1 - (a) Ubicazione del campo prove; (b) cartografia geologica (Sistema di coordinate: WGS 1984-UTM) (ISPRA Foglio 199).

Fig. 1 - (a) Location of the test site; (b) geological map (Coordinates refer to WGS 1984 -UTM Zone 32N projection). 
acquisita dal monitoraggio pre-durante e post trattamento nei numerosi casi reali (Fischanger, et al. 2007) e nei campi prova (Apuani et al 2011; Apuani et al. 2015) giustifica l'interesse ad un'intorno del punto di iniezione, che nelle condizioni sperimentali del campo prova è previsto dell'ordine di $1 \mathrm{~m}$ di raggio, come sarà verificato attraverso il rilevamento in trincea post iniezione.

i) Indagini pre trattamento: in analogia con quanto è consuetudine nelle procedure reali di trattamento condotte da GEOSEC s.r.l (Fischanger et al. 2009, www.geosec.it), viene innanzitutto eseguita un'indagine di tomografia di resistività elettrica (ERT) utilizzando un georesistivimetro IRIS SyscalPro. Nel campo sperimentale lo stendimento elettrico lineare di lunghezza $11.5 \mathrm{~m}$ è costituito da 24 elettrodi a spaziatura $0.5 \mathrm{~m}$ e con l'acquisizione di misure quadripolari di tipo Wenner e dipolo-dipolo, che consente una profondità di investigazione di circa $3 \mathrm{~m}$. Lo scopo principale delle indagini elettriche pre-trattamento è quello di verificare la distribuzione laterale e verticale delle unità stratigrafiche attraverso misure di resistività ed avere la garanzia che le indagini pre e post trattamento interessino lo stesso orizzonte geotecnico.

Per verificare i caratteri stratigrafici e la natura del terreno, già riconosciuti nello studio precedente, ed ottenere campioni rappresentativi, dopo aver rilevato lo stato del terreno in superficie e mappato l'andamento delle fratture visibili, sono stati eseguiti un sondaggio verticale (S1) fino a circa $-295 \mathrm{~cm}$ dal p.c. ed una perforazione ausiliaria (P1) che raggiunge -170 $\mathrm{cm}$, da cui sono stati prelevati, a profondità comprese tra 80 e $226 \mathrm{~cm}$, campioni da sottoporre alle analisi geotecniche di laboratorio. Le carote estruse sono state analizzate (ASTM
D2488) e misurati i valori di resistenza alla penetrazione (con penetrometro tascabile) e di resistenza al taglio non drenata (con scissometro tascabile).

E' stato poi realizzato uno scavo profondo $110 \mathrm{~cm}$ dal p.c., di circa $2 \times 3 \mathrm{~m}$ con un lato passante in prossimità della verticale P1. Lo scavo, eseguito con benna meccanizzata, consente di ottenere sezioni stratigrafiche esposte ed un piano di posa su cui eseguire prove di densità in situ con volumometro a sabbia e a membrana Figura 2, seguendo le procedure ASTM D155607 e ASTM D2167-08;

ii) Iniezioni con resine espandenti: l'iniezione viene eseguita con resine espandenti della famiglia commercialmente denominata MAXIMA ${ }^{\circledR}$ di GEOSEC ${ }^{\circledR}$ lungo la verticale ubicata al centro della sezione tomografica alla profondità di $-110 \mathrm{~cm}$ (Fig.2). La procedura GEOSEC prevede l'esecuzione di un preforo fino alla profondità di iniezione attraverso il quale, tramite apposito condotto, viene iniettata la miscela poliuretanica, $30 \mathrm{~kg}$ nello specifico studio.

MAXIMA® è un sistema poliuretanico bi-componente espandente a celle prevalentemente chiuse ed omogenee (AA. VV. 2001; Occhi 2014; Geosec Srl 2017), ove la schiuma è costituita da polimero solido per circa il $5 \%$ del volume e dalla restante parte di fase gassosa. La reazione chimica utilizza l'acqua come agente espandente nel pieno rispetto delle direttive comunitarie contro ogni forma di inquinamento atmosferico. I risultati dei test di compatibilità ambientale (D.lgs. 152/06 e s.m.i.) hanno dimostrato come i campioni di resina MAXIMA ${ }^{\circledR}$ siano risultati possedere tutti i valori di riferimento inferiori a quelli limite imposti (Istituto Giordano s.p.a 2017).

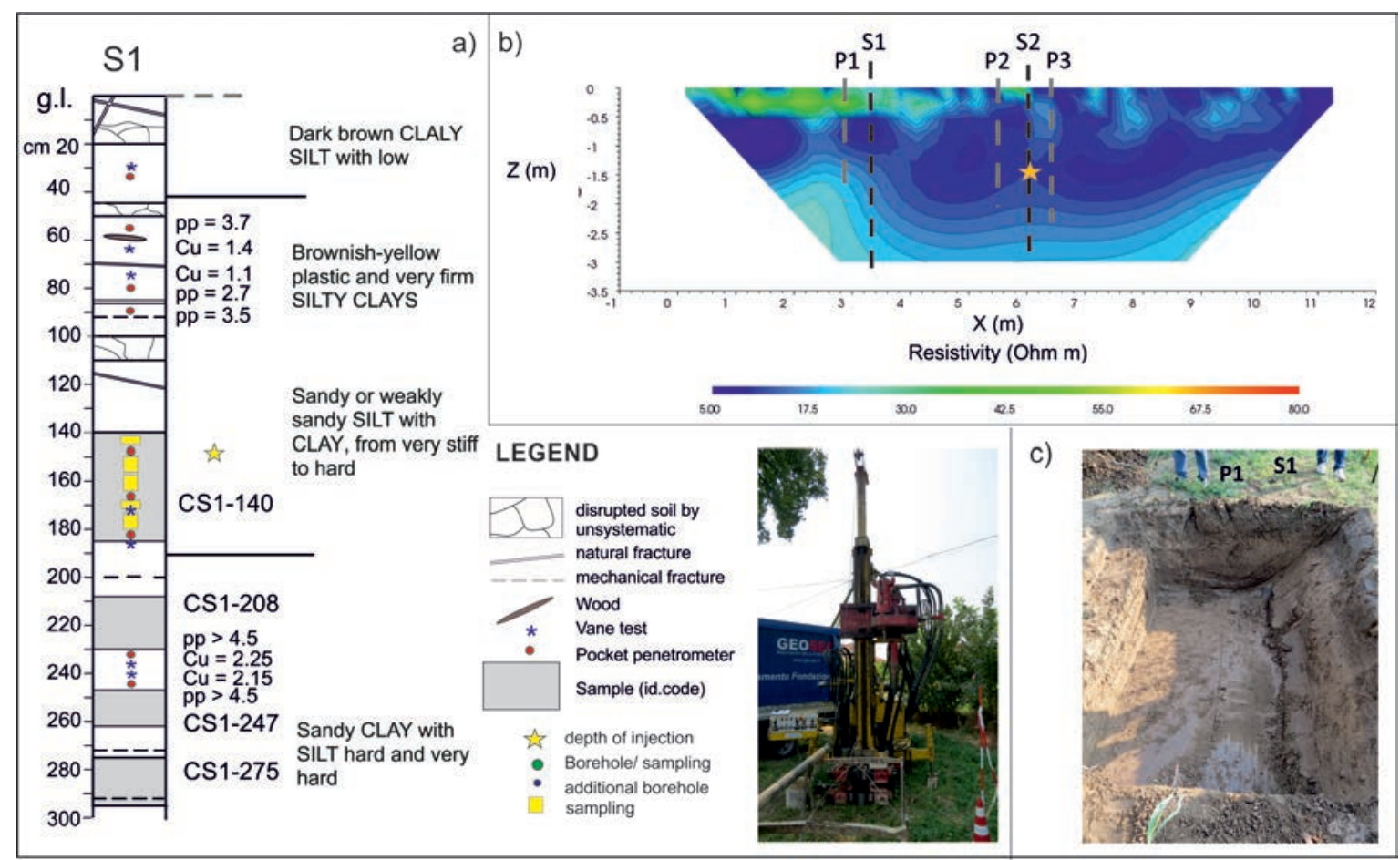

Fig. 2 - a) Stratigrafia del sondaggio S1; b) Tomografia elettrica Pre-iniezione; c) scavo di ispezione del terreno naturale (profondità -110cm).

Fig. 2 - a) Stratigraphy of the S1 probe; b) Electric pre-injection tomography; c) inspection pit in natural soil (depth-110cm). 
Il formulato chimico è caratterizzato da una reazione chimica irreversibile molto veloce rispetto ai comuni poliuretani industriali proprio per rimanere all'interno del volume significativo del terreno di fondazione. Terminata la reazione chimica, dopo circa qualche minuto, il sistema si stabilizza mantenendo uno stato inerte rigido e stabile, racchiudendosi in una pellicola di protezione continua ed impermeabile all'esterno, resistente all'azione di funghi, batteri e dei principali agenti chimici aggressivi. Numerosi test di laboratorio effettuati su differenti campioni di resina della famiglia MAXIMA® a diverse densità di formulazione attestano l'efficacia e la qualità del prodotto (elevate proprietà di resistenza, bassa deformabilità, (Geosec Srl 2017) e l'alta affidabilità per gli impieghi specifici di consolidamento e stabilizzazione dei terreni di fondazione e dei sottofondi di pavimentazioni cedute (Rittgers et al. 2010; d'Attoli 2016; Occhi et al 2017; Santarato et al. 2017; https://www.geosec. it/consolidamento-fondazioni/iniezioni-resine-espandenti/ resina/).

iii) Indagini post intervento: al termine del trattamento viene ripetuta un'ultima acquisizione di tomografia della resistività elettrica mantenendo invariato lo stesso impianto di acquisizione con successiva elaborazione delle variazioni percentuali indotte, sia di resistività che indirettamente di densità. Così facendo sarà dunque possibile individuare $i$ volumi di influenza del trattamento, a cui potranno essere riferire le variazioni dei parametri di conducibilità idraulica misurate in laboratorio.

Ad una distanza massima di circa $40 \mathrm{~cm}$ dalla verticale di iniezione, vengono poi eseguiti un sondaggio verticale (S2) fino a $-315 \mathrm{~cm}$ dal p.c. e due perforazioni ausiliarie (P2, P3) fino a $-200 \mathrm{~cm}$ dal p.c.. Campioni vengono prelevati a diverse profondità interessando gli orizzonti compresi tra -80 e -195 $\mathrm{cm}$ dal p.c.. Analogamente a quanto fatto nel dominio di terreno non trattato, viene realizzato uno scavo profondo 110 $\mathrm{cm}$ dal p.c., con un lato parallelo e distante circa $20 \mathrm{~cm}$ dal piano contenente le verticali P2, P3 e l'iniezione. Lo scavo fornisce l'opportunità unica, non ripetibile nei casi reali di trattamento, di osservare le modalità di propagazione delle resine nel terreno. Nello stesso tempo si ottengono sezioni stratigrafiche esposte che mettono a giorno gli effetti del trattamento di iniezione e forniscono un piano di posa su cui eseguire le prove di densità con volumometro a sabbia e a membrana.

iv) Prove di laboratorio: l'ultima fase ha previsto l'esecuzione di prove geotecniche di laboratorio su campioni prelevati in condizioni naturali: analisi granulometriche, limiti di Atterberg, determinazioni dei pesi di volume, del peso specifico e delle condizioni di umidità naturale; prove di permeabilità in cella triassiale e prove edometriche. Anche sui terreni prelevati nell'intorno del trattamento sono state eseguite prove di permeabilità in cella triassiale. Una sintesi delle prove eseguite e delle specifiche procedurali adottate è riportata in Tab.1.

\section{Prove di permeabilità in cella triassiale}

Allo scopo di valutare gli effetti del trattamento sulle proprietà di conducibilità idraulica, prove di permeabilità in cella triassiale sono state eseguite, secondo normativa BS 1377-6:1990, su campioni di terreno naturale e trattato. La prova di permeabilità utilizza la cella triassiale nella quale un campione cilindrico viene saturato, consolidato (Fig. 3a) e sottoposto a flusso verticale imponendo una differenza di pressione di poro $(\mathrm{p} 1-\mathrm{p} 2=\Delta \mathrm{p})$ tra le basi superiore ed inferiore del campione. Il coefficiente di permeabilità è calcolato dalla curva flusso cumulato $(\mathrm{ml})$ vs. tempo ( $\mathrm{min}$ ) al raggiungimento di una condizione di flusso stazionario (Fig. 3b)

Sebbene nelle prove di permeabilità in cella triassiale gli stati tensionali siano di tipo isotropo, condizione rara nei casi reali di utilizzo delle resine ai fine del consolidamento di terreni di fondazione, si è scelto di prediligere queste alle prove edometriche che, pur simulando lo stato anisotropo in situ, sono finalizzate alla determinazione delle proprietà geotecniche di consolidazione. Tali prove consentono di

Tab.1 - Elenco delle prove geotecniche eseguite: riferimenti alle procedure standard e numero di prove. .

Tab.1 - List of the performed geotechnical tests: standard references and number of tests for natural and traitted soil.

\begin{tabular}{|c|c|c|c|}
\hline \multirow{2}{*}{$\begin{array}{c}\text { Tipologia di prova } \\
\text { test type }\end{array}$} & \multirow{2}{*}{$\begin{array}{l}\text { Normativa di } \\
\text { riferimento } \\
\text { Standards }\end{array}$} & $\begin{array}{c}\text { Terreni naturali } \\
\text { natural soil }\end{array}$ & $\begin{array}{c}\text { Terreni tratttati } \\
\text { treatted soil }\end{array}$ \\
\hline & & \multicolumn{2}{|c|}{$\mathbf{N}^{\circ}$ di prove $-n^{\circ}$ of test } \\
\hline Prove di densità con volumometro a sabbia & ASTM D 1556 & 1 & 2 \\
\hline Prove di densità con volumometro a membrana & ASTM D 2167 & 4 & 1 \\
\hline Estrusione e descrizione dei campioni fustellati, misura $\mathrm{Pp}$ e $\mathrm{Cu}$ & ASTM D2488 & & \\
\hline Analisi granulometriche & ASTM E112-96 & 8 & - \\
\hline Determinazione degli indici di consistenza & ASTM D4318 & 6 & - \\
\hline Determinazione del peso specifico & ASTM D854 & 5 & - \\
\hline Determinazione dei pesi di volume & ASTM D2937 & 10 & 10 \\
\hline Determinazione del contenuto d'acqua naturale & ASTM D2216 & 13 & 10 \\
\hline Prove di conducibilità in cella triassiale (provini indisturbati) & BS - 1377-6:1990 & 12 & 15 \\
\hline Prove edometriche (provini indisturbati) & BS - 1377-5:1990 & 2 & 1 \\
\hline
\end{tabular}


a)
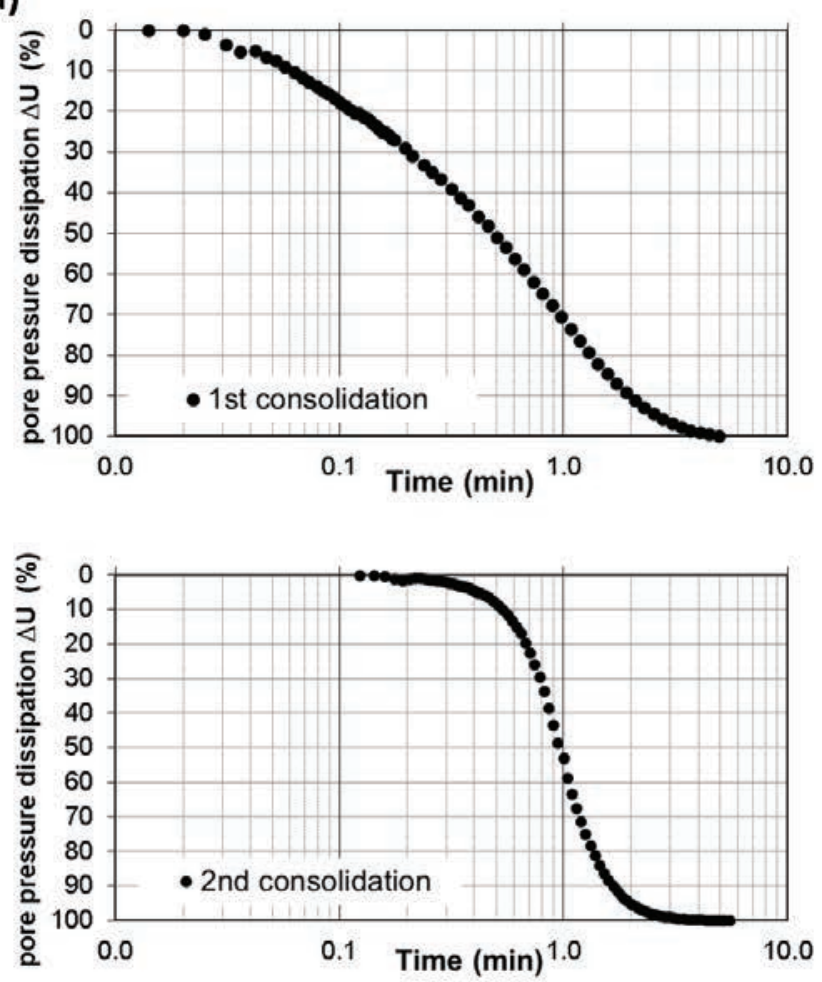

b)
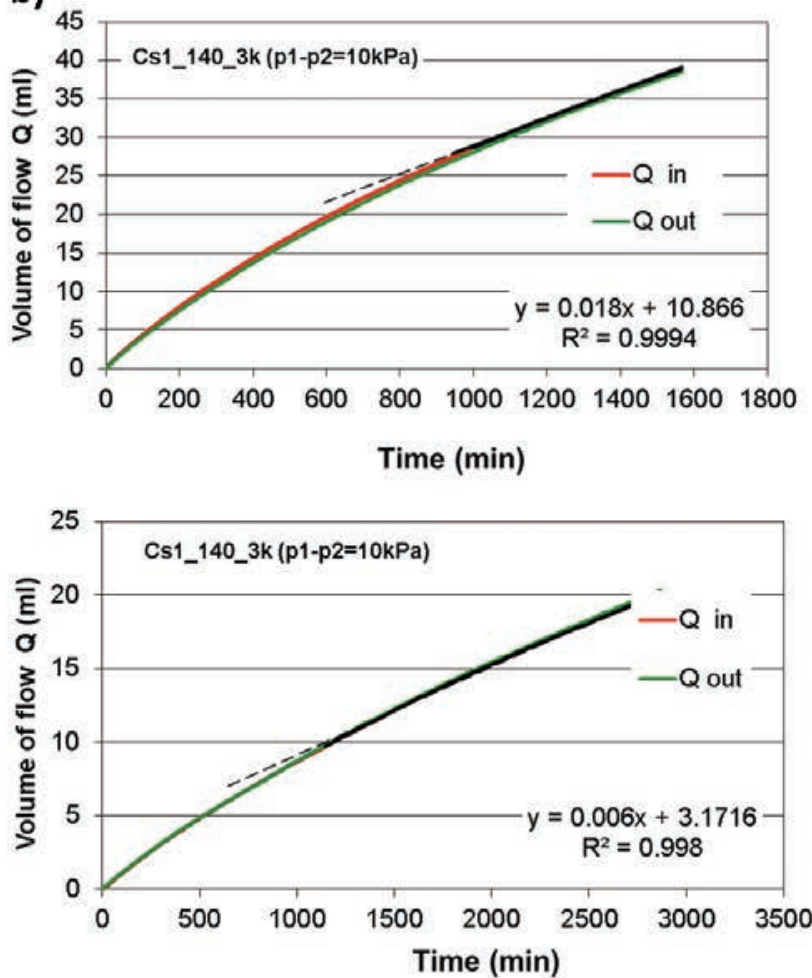

Fig. 3 - a) Esempio curve di consolidazione e (b) curva di flusso cumulato vs tempo per la determinazione della permeabilità in cella triassiale alla fine di ciascuna fase di consolidazione

Fig. 3 - a) Example of consolidation curve and (b) cumulative flow vs. time curve in triaxial cell permeability test at the end of each consolidation phase..

calcolare la permeabilità solo indirettamente, attraverso la misura dei cedimenti e deducendo il comportamento alla filtrazione senza la diretta misura di u. Al contrario le prove di permeabilità in cella triassiale sono esplicitamente formulate allo scopo. Prove edometriche sui terreni naturali sono state comunque eseguite nel presente studio allo scopo di definire il grado di sovraconsolidazione del terreno naturale come supporto a commento delle proprietà idrogeologiche e fisiche misurate.

Dunque, provini indisturbati di diametro ed altezza pari a $50 \mathrm{~mm}$ sono stati ottenuti mediante campionamento con fustella dalla carota di sondaggio del diametro di 70 , seguendo le procedure dettate dalla normativa BS1377-1 (punto 8 metodo b). I campioni, prelevati a profondità comprese tra -145 e $-190 \mathrm{~cm}$, si trovano in situ in condizioni di forzi efficaci $\sigma^{\prime}{ }_{v o}$ comprese tra 28 e $30 \mathrm{kPa}$. Hanno indice dei vuoti iniziali eo compresi tra 0.53-0.55 nel caso di terreni naturali e compresi tra $0.51-0.63$ nel caso dei campioni prelevati in prossimità del trattamento. E' stato scelto di eseguire le misure imponendo condizioni di sforzi efficaci $\sigma_{3}^{\prime}=160 \mathrm{kPa}$ e $360 \mathrm{kPa}$, senza discostarsi eccessivamente dalle condizioni tensionali dei primi metri di terreno sotto l'ipotetico carico verticale di un edificio. Le prove hanno perciò previsto una fase di saturazione, una prima consolidazione fino a $\sigma_{3}^{\prime}=160 \mathrm{kPa}$, raggiunta con unico gradino, l'applicazione di un gradiente idraulico tra le basi (per $\Delta \mathrm{p}=10 \mathrm{kPa}$ ) e la misura delle portate cumulate, dunque il calcolo di un primo valore di permeabilità $\mathrm{K}(160$-10); dopodiché un secondo gradino di consolidazione ha portato il campione alla condizione $\sigma_{3}^{\prime}=360 \mathrm{kPa}$ e la permeabilità $\mathrm{K}(360-10)$ nuovamente calcolata per applicazione di una differenza di pressione $\Delta \mathrm{p}=10 \mathrm{kPa}$. La fase di scarico fino alla stessa tensione $\sigma_{3}^{\prime}=160 \mathrm{kPa}$ è seguita da una nuova misura della permeabilità $\mathrm{K}(160 \mathrm{sc}-10)$. In alcuni casi si è verificato l'effetto indotto dall'applicazione di una diversa differenza di carico, applicando $\Delta \mathrm{p}=20 \mathrm{kPa}$. Le condizioni di prova in termini di stato tensionale a fine consolidazione e gradiente idraulico sono rappresentate in Fig. 4.

\section{Risultati \\ Caratteristiche litostratigrafiche $e$ di resistività elettrica, identificazione geotecnica e proprietà indice del terreno naturale}

Le informazioni sulla natura del sottosuolo, sulla stratigrafia e sull'assortimento granulometrico, derivano dall'analisi congiunta delle stratigrafie di sondaggio, dalle osservazioni lungo le pareti di scavo, dalle indagini geofisiche e dalle prove di identificazione condotte sui campioni naturali in laboratorio. Le indagini confermano i caratteri litostratigrafici riscontrati ad una decina di metri di distanza e documentati in Apuani et al, 2015.

Nel volume di sottosuolo investigato, fino alla profondità di circa $3 \mathrm{~m}$, comprensivo del terreno dedicato alle indagini pre e post-trattamento si riconoscono caratteri stratigrafici continui 


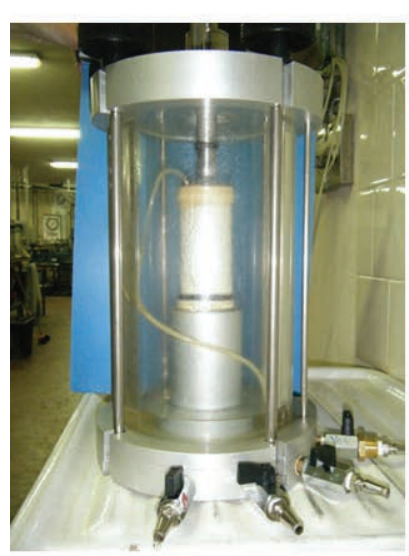

Loading and unloading phases

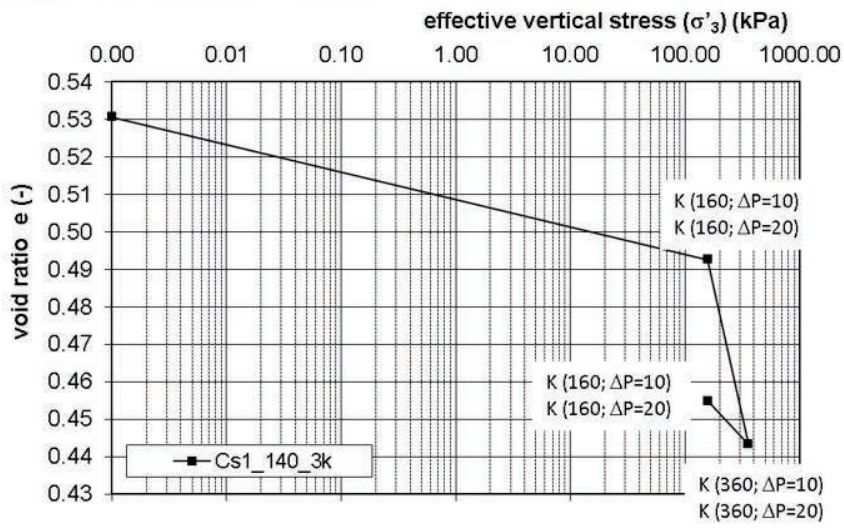

Fig. 4 - a) Dettaglio fotografico della cella triassiale per le prove di permeabilità. b) Esempio delle condizioni di prova in cella triassiale: sforzi efficaci $\left(\sigma_{3}^{\prime}\right)$ al termine della consolidazione vs indice dei vuoti (e) e differenza di pressione $(\Delta \mathrm{p})$ che genera filtrazione.

Fig. 4 - Permeability test in triaxial cell: a) image of the triaxial cell for Permeability test. b) Graphical example of the performed test steps: effective vertical stress ( $\left.\sigma_{3}^{\prime}\right)$ at the end of consolidation vs void ratio (e) at different pore pressure difference $(\Delta p)$.

ed omogenei. Si tratta di terreni coesivi omogenei con modeste locali variazioni verticali nella componente argillosa e sabbiosa; con la profondità si riscontra un generale incremento della consistenza e coesione, misurate con penetrometro e scissometro tascabili. Complessivamente si distinguono alcuni orizzonti caratteristici. (1) Il primo, da p.c. a circa -40 $\mathrm{cm}$, consiste in terreno areato, limo argilloso di colore bruno scuro, granulometria omogenea, consistente con resistenza alla penetrazione $\mathrm{pp}<2.9 \mathrm{~kg} / \mathrm{cm}^{2}$, medio-bassa plasticità e coesione non drenata $\mathrm{Cu}=0.75 \mathrm{~kg} / \mathrm{cm}^{2}$. In genere questo orizzonte è intensamente fratturato e localmente disgregato in zolle; ha resistività elettrica $\rho$ intorno a $30 \omega / \mathrm{m}$ con valori fino a $50 \omega / \mathrm{m}$ rilevabili in corrispondenza di porzioni di terreno con fessure superficiali (Fig. 2b). (2) Il secondo, fino alla profondità di circa $90 \mathrm{~cm}$, è costituito da argilla limosa che si distingue dal primo orizzonte per il colore più chiaro bruno-giallastro, l'assenza di macro fratture, valori medi di resistività $\rho$ compresi tra $5 \div 10 \omega / \mathrm{m}$, maggior consistenza $\left(3.3<\mathrm{pp}<3.7 \mathrm{~kg} / \mathrm{cm}^{2}\right)$ e coesione non drenata $(1.1<\mathrm{Cu}<1.5 \mathrm{~kg} /$ $\mathrm{cm}^{2}$ ). Apparati radicali di diametro anche centimetrico sono presenti fino a circa $80 \mathrm{~cm}$ dal p.c. Entrambe gli orizzonti 1) e 2) sono stati rimossi dagli scavi che espongono in piano l'unità sottostante. A profondità superiori a $90 \mathrm{~cm}$ il terreno può considerarsi piuttosto omogeno; la distinzione geotecnica tra orizzonti non è particolarmente marcata, tuttavia possiamo annotare da $90 \mathrm{~cm}$ a circa $180 \mathrm{~cm}$ l'orizzonte (3) costituito da limo con argilla debolmente sabbioso, di colore marrone, da molto consistente a duro $\mathrm{pp}>3.7 \mathrm{~kg} / \mathrm{cm}^{2}$, coesione non drenata variabile localmente $1.5<\mathrm{Cu}<2.3 \mathrm{~kg} / \mathrm{cm}^{2}$. (4) $\mathrm{Da} 190$ a $300 \mathrm{~cm}$ il terreno è argilla con limo sabbiosa con inclusi millimetrici in aumento con la profondità, di consistenza dura $\mathrm{pp}>4.5 \mathrm{~kg} / \mathrm{cm}^{2}$, coesione non drenata $2.15<\mathrm{Cu}<2.4 \mathrm{~kg} /$ $\mathrm{cm}^{2}$. Tale variazione granulometrica è riscontrabile anche nel profilo verticale di resistività che mostra un debole e graduale incremento in profondità.

Poiché l'iniezione di resina è eseguita nell'orizzonte (3) alla profondità di $150 \mathrm{~cm}$ dal p.c., prevedendone gli effetti in un volume distante fino a $1 \mathrm{~m}$ dal centro di iniezione, tutte le prove eseguite a fondo scavo interessano l'orizzonte (3) e le prove geotecniche sono concentrate sui campioni prelevati alle profondità potenzialmente coinvolte dal trattamento tra -120 $\mathrm{cm} \mathrm{e}-230 \mathrm{~cm}$ dal p.c..

Si tratta di limi con argilla, debolmente sabbiosi, ovvero $\mathrm{CL}$ (localmente $\mathrm{CH}$ ) secondo classificazione Unified Soil Classification System USCS (ASTM D2487-11), disuniformi o molto disuniformi (Fig. 5a) a media plasticità (limite liquido $\mathrm{WL}=43 \div 54 \%$ e indice plastico $\mathrm{IP}=21 \div 31 \%$ ) (Fig. 5 b) inattive (Fig. 5c), in condizioni di umidità naturale $\mathrm{W}=15 \div 23 \%$, molto consistenti o localmente dure (AGI Raccomandazioni, 1997; BS:5930), coerentemente con lo studio precedente (Apuani et al.2015) di cui si riportano per confronto i valori, sia nelle figure che nel seguito del testo.

Prove di densità eseguite in situ mediante volumometro a sabbia (ASTM D1556-07) e a membrana (ASTM D2167) e in laboratorio con metodo del cilindro sagomato tagliente (ASTM D2937-99), fornisco valori medi di peso di volume secco $\gamma_{d}=15.7 \div 17.4 \mathrm{kN} / \mathrm{m}^{3}\left(\gamma_{d}=16.8 \pm 1.2 \mathrm{kN} / \mathrm{m}^{3}\right.$ nello studio precedente) con porosità calcolata $\mathrm{n}=32 \div 39 \%(\mathrm{n}=34 \pm 5 \%)$. Il peso di volume naturale varia nel campo $\gamma_{0}=19.0 \div 20.5 \mathrm{kN} /$ $\mathrm{m}^{3}\left(\gamma_{0}=19.7 \pm 0.9 \mathrm{kN} / \mathrm{m}^{3}\right)$.

\section{Ispezione diretta dei fronti scavo intorno all'iniezione e proprietà indice del terreno trattato}

Le operazioni di scavo hanno esposto il terreno consolidato con iniezioni di resina Fig.6a, rimuovendo progressivamente il terreno in prossimità dell'iniezione fino $\mathrm{a}-110 \mathrm{~cm}$ appena al di sopra del centro di iniezione $(-150 \mathrm{~cm})$. L'operazione offre la possibilità di osservare direttamente le modalità di migrazione delle resine iniettate, opportunità che non si presenta nei casi reali in cui il trattamento interessa terreni di fondazione, riconoscendo direzioni preferenziali e distanze raggiunte dal trattamento Fig.6b, oltre a fornire il piano di esecuzione per le misure di densità, di resistenza alla penetrazione e coesione non drenata. 
a)

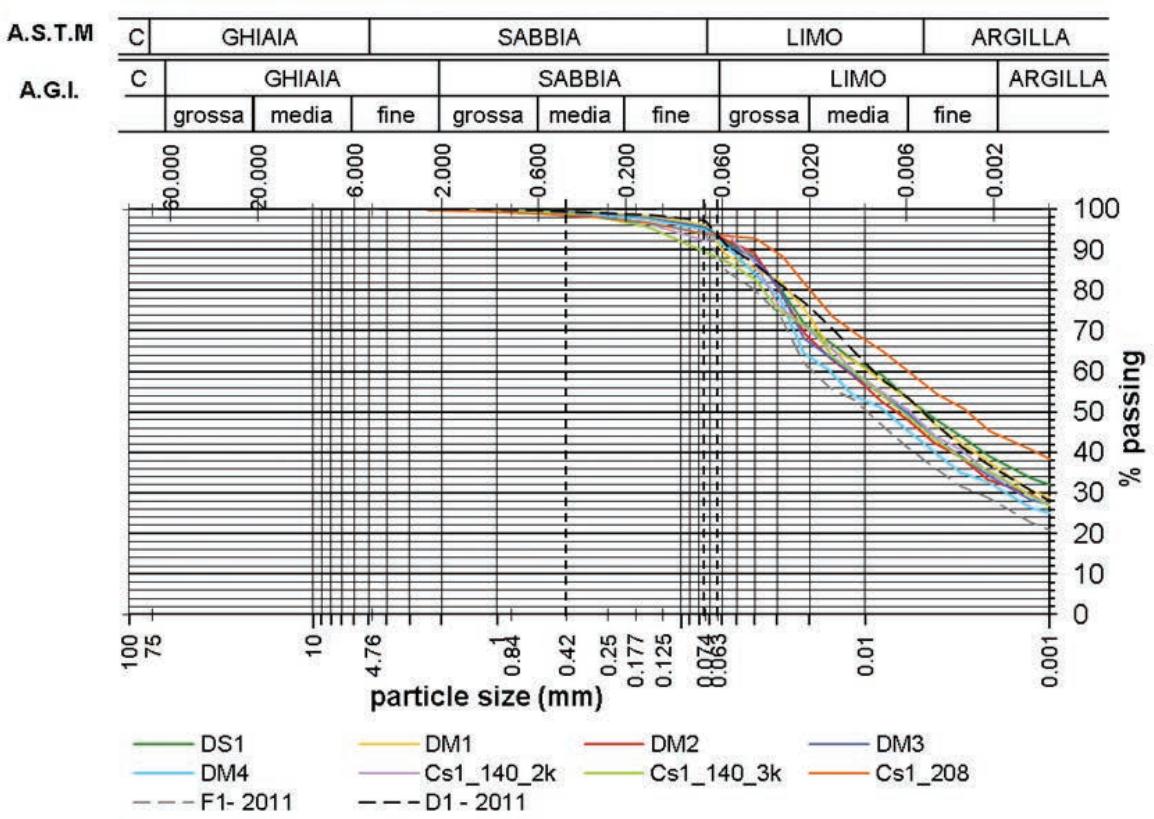

b)

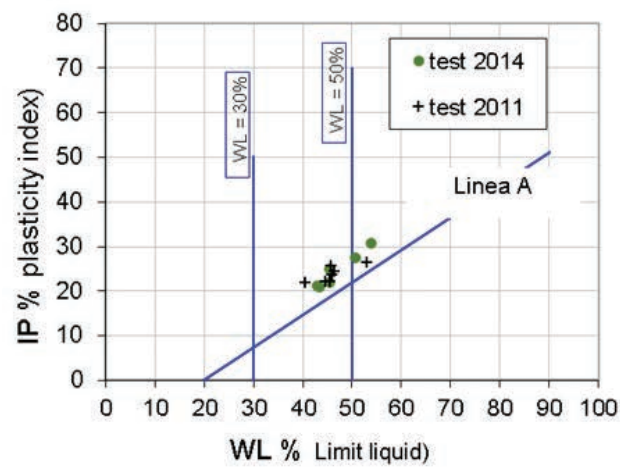

c)

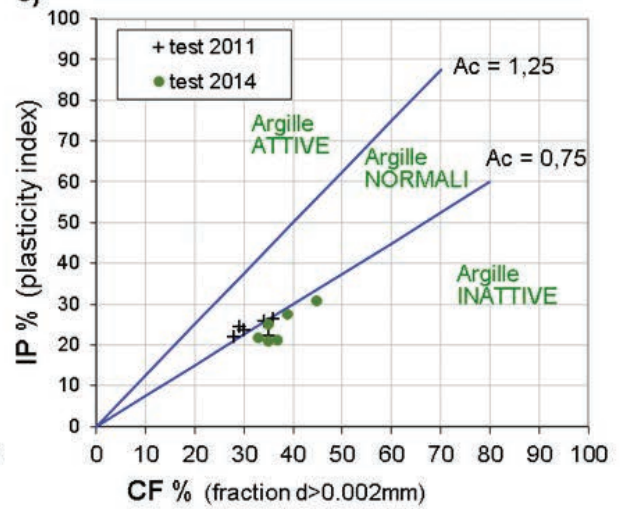

Fig. 5 - a) Curve granulometriche caratterizzanti il terreno tra -110 e $-220 \mathrm{~cm}$. Per confronto le curve tratteggiate grigie rappresentano gli estremi del fuso granulometrico rappresentativo della profondità -140 $\mathrm{cm}$ ottenuto dal precedente test del 2011 nello stesso sito (Apuani et al. 2015); b) carta di plasticità; c) carta di attività.

Fig. 5 - a) Particle size curves related to the soil between -110 and $-230 \mathrm{~cm}$. By comparison, the gray dashed curves represent the extremes of the granulometric range representative of the depth $-140 \mathrm{~cm}$, and obtained from the previous work. (Apuani et al. 2015) in the same site; b) plasticity chart; c) activity chart.
Landamento e la distribuzione delle vene è confrontata con le condizioni di fessurazione e di debolezza individuate durate il rilevamento pre-trattamento dalla superficie e nello scavo attiguo di terreno naturale. Sebbene si tratti di una sola iniezione, si confermano le osservazioni riportate nello studio precedente (Apuani et al 2011; Apuani et al. 2015): la resina ha compattato il terreno riempiendo fratture preesistenti ed orizzonti deboli, riconosciuti nel rilevamento pre-iniezione di superficie e/o nello scavo attiguo non trattato, ed esercitando la propria pressione di espansione. È ben evidente (Fig. 6a,b) come la presenza di vuoti o canali preesistenti costituiscano la via di fuga preferenziale per la distribuzione delle resine; la resina tende a seguire e occupare volumi altrimenti deboli, in genere a maggior contenuto idrico. Lo spessore delle vene occupate dalla resina raggiunge il centimetro di larghezza e la distanza di migrazione dal punto di iniezione supera il metro di lunghezza. Le vene interessano una porzione di terreno rilevante anche al di sopra e al di sotto del punto di iniezione. Si viene inoltre a costituire un reticolo capillare di resina che va a "rinforzare" il terreno nelle adiacenze. Le operazioni di scavo, eseguite con la stessa macchina escavatrice dello scavo pre trattamento, avevano già fatto apprezzare sia l'incremento di resistenza generale del terreno trattato, sia quanto le vene di resina rappresentassero per l'escavatore un orizzonte "indurito."

Anche alla scala del campione macroscopico i campioni prelevati nel sondaggio si presentano con diverso grado di "impregnazione" di resina Fig. 6c,d: le resine possono presentarsi in vene, in forma di micro-capillari o non essere riconoscibili a scala macroscopica. I parametri di resistenza alla penetrazione pp e di coesione non drenata $\mathrm{Cu}$ si confermano alti, spesso oltre il fondo scala strumentale, come già misurato nel terreno naturale $\left(\mathrm{pp}>4.5 \mathrm{~kg} / \mathrm{cm}^{2}\right)$.

Le prove geotecniche eseguite in sito e in laboratorio sui terreni trattati forniscono i seguenti valori: peso di volume secco $\gamma_{\mathrm{d}}=16.70 \pm 0.70 \mathrm{kN} / \mathrm{m}^{3}$ (valore medio \pm deviazione standard) e peso di volume naturale $\gamma_{o}=19.63 \pm 0.28 \mathrm{kN} / \mathrm{m}^{3}$, con porosità calcolata $\mathrm{n}=35.8 \pm 1.9 \%$. Il contenuto d'acqua in condizioni di saturazione risulta $\mathrm{W}_{\mathrm{sat}}=27.5 \pm 2.8 \%$.

\section{Variazioni del comportamento resistivo post trattamento}

Le elaborazioni delle misure per la determinazione dei modelli di resistività del sottosuolo (inversioni) sono state eseguite con l'ausilio del software ERTLab64TM che utilizza un algoritmo di inversione ai minimi quadrati con vincolo 


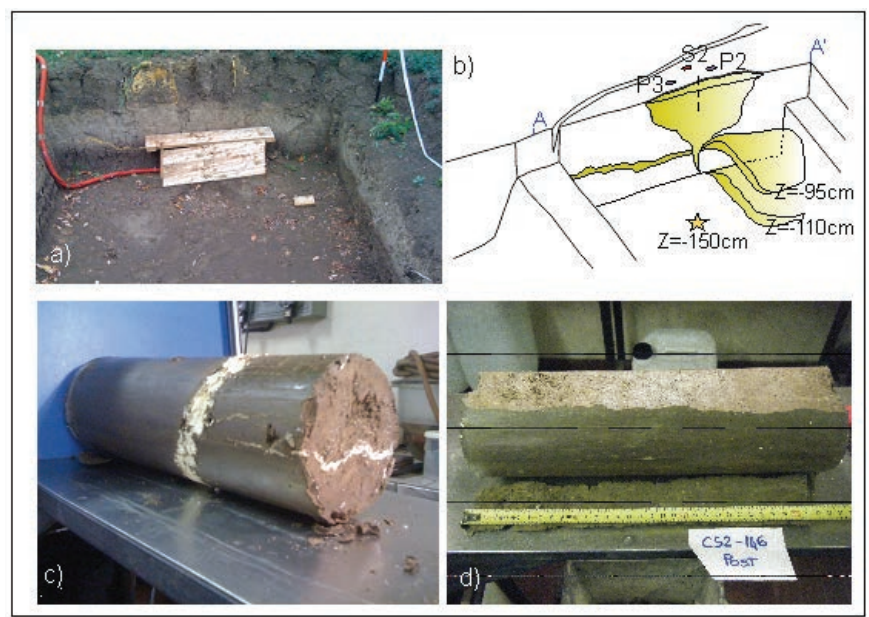

Fig. 6 - a) Campo prova: scavo post trattamento. Immagine delle pareti di scavo in prossimità dell'iniezione; b) schizzo rappresentante la distribuzione delle resine nel volume di terreno rimosso dalla scovo nell'intorno dell'iniezione. c) e d) Materiali estrusi dalle fustelle, differenti per modalità ed entità di impregnazione da resine: campione $\mathrm{CP} 3-80$ con vene centimetriche (c), campione CS2-146 con compattazione e impregnazione diffusa (d).

Fig. 6 - Test site: after treatment pit: a) view of the vertical walls of excavation, near the injection; b) Sketch of the distribution of the resins in the volume of soil removed. $c$ ) and d) Samples extruded from the after-treatment boreholes; different mode and extent of resin impregnation: sample CP3-80, with centimetric resin veins (c); sample CS2-146, uniformly impregnated $(d)$.

di regolarità tra celle adiacenti (Morelli e LaBrecque 1996). L'acquisizione di misure reciproche ha consentito di stimare una deviazione standard media sulle misure acquisite inferiore allo $0.5 \%$. Le due acquisizioni pre e post-iniezione sono state eseguite a distanza di 1 ora e 40 minuti, nelle medesime condizioni meteo, in assenza di precipitazioni e in condizioni di omogeneità della temperatura. Le indagini elettriche forniscono le sezioni tomografiche di Fig. 7
Il modello elettrico post-iniezione manifesta un deciso incremento lungo la verticale di iniezione. In particolare nel caso specifico di una singola iniezione eseguita su terreno scarico l'incremento resistivo si distribuisce in un volume di raggio stimato di circa $1 \mathrm{~m}$ nell'intorno superiore del centro di iniezione. Tale risultanze sono coerenti con la distribuzione delle resine osservata durante le fasi di scavo (Fig. 6b). La presenza di piani sub orizzontali costituiti dalle resine in espansione testimonia il crearsi di condizioni di flusso impedito verso l'alto, che si manifesta in un non incremento delle resistività nel volume inferiore all'iniezione.

I volumi di terreno influenzati dal trattamento sono dipendenti da quantitativi di resina iniettati e la geometria del corpo è controllata prevalentemente dalla natura e stratigrafia del mezzo geologico.

\section{Valori di conducibilità Idraulica in terreni naturali $e$ trattati}

Dall'analisi delle curve di flusso cumulato vs. tempo, registrate per ciascun provino e in ciascuna delle condizioni di sollecitazione imposte e descritte al paragrafo 2.3 e in Fig. 3, la conducibilità idraulica verticale è calcolata al raggiungimento della condizione di flusso stazionario secondo l'equazione dettata dalle norme procedurali (BS1377-6)

$$
K=\frac{1.63 q L}{A\left[\left(p_{1}-p_{2}\right)-p_{c}\right]} \cdot R t \cdot 10^{-4}[\mathrm{ml} / \mathrm{min}]
$$

dove $q=$ portata media attraverso il provino $\left(\mathrm{cm}_{3} / \mathrm{min}\right)$, $\mathrm{L}=$ percorso idraulico $(\mathrm{mm}) ; \mathrm{A}=$ sezione del provino $\left(\mathrm{mm}^{2}\right)$, $\left(p_{1}-p_{2}\right)=$ differenza di pressione applicata alla base e alla sommità del provino $(\mathrm{kPa}), p_{c}=$ perdita di pressione $(\mathrm{kPa})$ alla
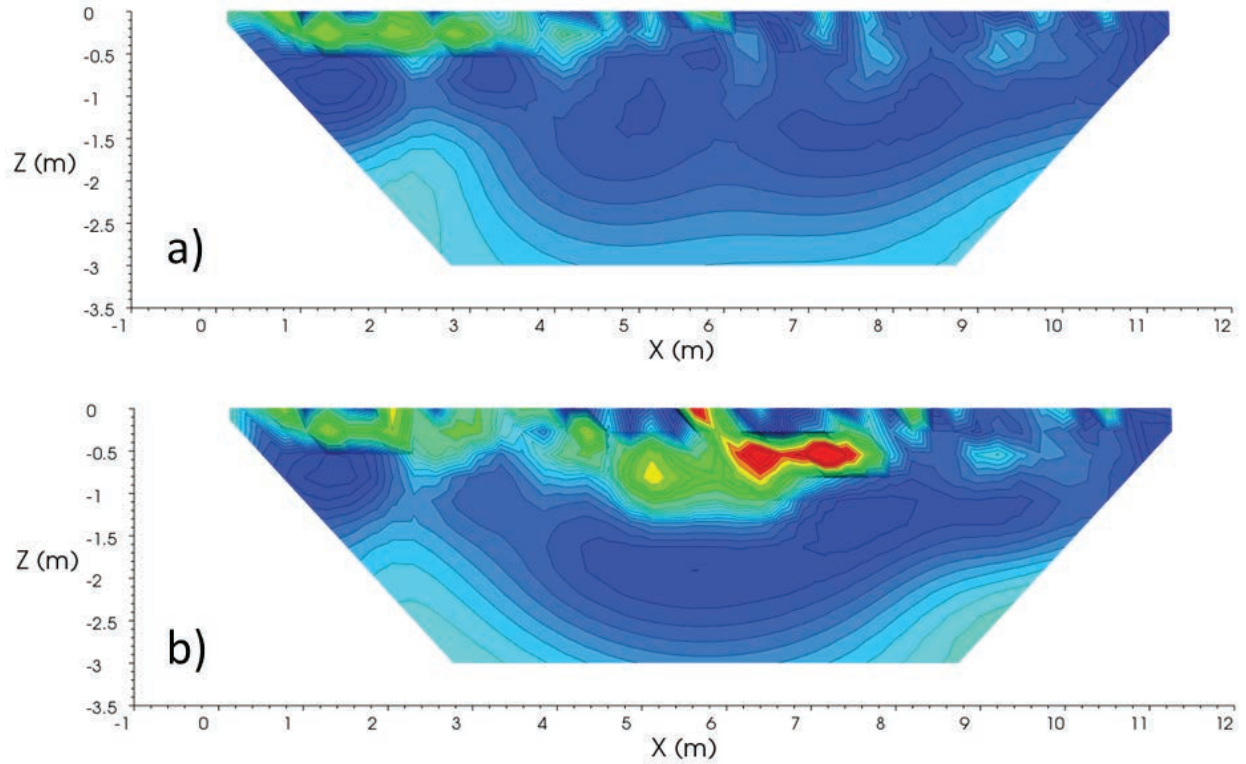

\begin{tabular}{ccccccc}
\multicolumn{8}{c}{ Resistivity (Ohm m) } \\
\hline 5.00 & 17.5 & 30.0 & 42.5 & 55.0 & 67.5 & 80.0
\end{tabular}

Fig. 7 - Sezioni di resistività elettrica: a) pre trattamento, b) post trattamento.

Fig. 7 - Electrical resistivity vertical sections: a) before injection, b) after injection. 
portata $q$, ottenuta da calibrazione; $R t=$ fattore di correzione funzione della temperatura.

La Tab.2 riporta tutti i risultati ottenuti specificando l'identificativo del campione (la cui profondità è definita nel nome - es CS1_140_2k z=140 cm) e le diverse condizioni di sollecitazione, mentre la Tab.3 riassume i valori medi calcolati.

La norma di riferimento non impone il calcolo della conducibilità idraulica dalle curve di consolidazione che precedono ciascuna delle successive fasi di prova (curva portata vs tempo). Dalle due condizioni sono infatti attesi valori diversi, come in genere avviene per tutte le grandezze ottenute con diverse condizioni al contorno. Tuttavia in Tab. 2 si riportano anche i valori di conducibilità idraulica di consolidazione che offrono, insieme al comportamento deformativo registrato in fase di consolidazione, un ulteriore dato di confronto per la valutazione degli effetti del trattamento.

Nel presentare i risultati va innanzitutto sottolineato che i valori di permeabilità misurati per i terreni analizzati $(\mathrm{CL}$, localmente $\mathrm{CH}$ ) sono estremamente bassi, in alcuni casi al limite della capacità strumentale, con valori di k, convenzionalmente ottenuti dalle curve di flusso, compresi tra $2.30 \mathrm{E}-09 \mathrm{~m} / \mathrm{s}$ e $2.32 \mathrm{E}-07 \mathrm{~m} / \mathrm{s}$ nei terreni naturali e compresi tra $1.74 \mathrm{E}-10 \mathrm{~m} / \mathrm{s}$ e $6.19 \mathrm{E}-09 \mathrm{~m} / \mathrm{s}$ per campioni interessati dal trattamento.

Nonostante ciò alcune significative differenze tra i valori pre e post trattamento si possono riconoscere. Focalizzando l'attenzione sui valori ottenuti dalle curve di flusso, i bassi valori di $\mathrm{k}$ calcolati per $\mathrm{i}$ campioni naturali (valor medio kpre-trattamento $=5.29 \mathrm{E}-08$ ) sono coerenti con i caratteri fisico-meccanici del terreno in esame e compatibili con quanto noto in letteratura per argille. Va inoltre considerato che prove edometriche condotte nel presente studio su provini indisturbati ottenuti dagli stessi campioni e rappresentativi dello stesso orizzonte stratigrafico, hanno confermato che si tratta di terreni molto consistenti (con Indice di consistenza Ic $>1$ ), apparentemente sovraconsolidati (forse per fenomeni di essicazione in situ) con rapporto di sovraconsolidazione $6.8>\mathrm{OCR}>7.8$.

Tab. 2 - Sintesi delle condizioni di prova e valori di permeabilità ottenuti in cella triassiale. Confronto dei valori in terreni naturali e trattati.

Tab. 2 - Synthesis of the test conditions and permeability values obtained in triaxial cell. Comparison of values in natural (pre) and treated (post) soils.

\begin{tabular}{|c|c|c|c|c|c|c|c|}
\hline Campione & $\begin{array}{c}\sigma_{3}^{\prime} \\
{[\mathrm{kPa}]}\end{array}$ & e & $\begin{array}{c}\mathrm{K} \text { cons. } \\
{[\mathrm{m} / \mathrm{s}]}\end{array}$ & $\begin{array}{c}\Delta \mathrm{p} \\
{[\mathrm{kPa}]}\end{array}$ & $\begin{array}{c}\mathrm{k} \\
{[\mathrm{m} / \mathrm{s}]}\end{array}$ & $\begin{array}{c}\Delta \mathrm{p} \\
\lfloor\mathrm{kPa}\rfloor\end{array}$ & $\begin{array}{c}\mathrm{k} \\
{[\mathrm{m} / \mathrm{s}]}\end{array}$ \\
\hline \multicolumn{8}{|c|}{ PRE - trattamento } \\
\hline \multirow{3}{*}{ Cs1_140_2k } & 160 & 0.54 & $2.23 \mathrm{E}-07$ & 10 & $2.32 \mathrm{E}-07$ & 20 & $9.50 \mathrm{E}-08$ \\
\hline & 360 & 0.52 & $3.70 \mathrm{E}-07$ & 10 & $7.95 \mathrm{E}-08$ & 20 & $6.66 \mathrm{E}-08$ \\
\hline & 159.9 & 0.53 & & 10 & $6.86 \mathrm{E}-08$ & 20 & $6.14 \mathrm{E}-08$ \\
\hline \multirow{3}{*}{ Cs1_140_3k } & 160 & 0.49 & $1.10 \mathrm{E}-07$ & 10 & $1.23 \mathrm{E}-08$ & 20 & $6.49 \mathrm{E}-09$ \\
\hline & 360.1 & 0.44 & $3.20 \mathrm{E}-08$ & 10 & $4.92 \mathrm{E}-09$ & 20 & $2.49 \mathrm{E}-09$ \\
\hline & 160 & 0.46 & & 10 & $3.25 \mathrm{E}-09$ & 20 & $2.30 \mathrm{E}-09$ \\
\hline \multicolumn{8}{|c|}{ POST- trattamento } \\
\hline \multirow{3}{*}{ Cs2_183_1k } & 139.8 & 0.59 & $9.70 \mathrm{E}-08$ & 10 & $3.04 \mathrm{E}-09$ & & \\
\hline & 160 & 0.59 & & 10 & $2.37 \mathrm{E}-09$ & & \\
\hline & 140 & 0.59 & & 10 & $2.20 \mathrm{E}-09$ & & \\
\hline \multirow{3}{*}{ Cs2_146_2k } & 160 & 0.48 & $4.40 \mathrm{E}-08$ & 10 & 6.19E-09 & 20 & 3.49E-09 \\
\hline & 359.9 & 0.46 & $1.40 \mathrm{E}-08$ & 10 & $3.36 \mathrm{E}-09$ & 20 & $1.46 \mathrm{E}-09$ \\
\hline & 159.9 & 0.47 & & 10 & $1.95 \mathrm{E}-09$ & 20 & $1.61 \mathrm{E}-09$ \\
\hline \multirow{3}{*}{ Cs2_146_3k } & 159.9 & 0.47 & $1.80 \mathrm{E}-09$ & 10 & $4.42 \mathrm{E}-10$ & 20 & 3.49E-10 \\
\hline & 359.3 & 0.44 & $4.90 \mathrm{E}-10$ & 10 & $3.05 \mathrm{E}-10$ & 20 & $1.86 \mathrm{E}-10$ \\
\hline & 160 & 0.45 & & 10 & $2.52 \mathrm{E}-10$ & 20 & $1.74 \mathrm{E}-10$ \\
\hline
\end{tabular}

Tab. 3 - Valori medi di permeabilità ottenuti in cella triassiale. Confronto dei valori in terreni naturali (pre) e trattati (post).

Tab. 3 - Average permeability values obtained in triaxial cell. Comparison of values in natural (pre) and treated (post) soils.

\begin{tabular}{|c|c|c|c|c|}
\hline & \multirow{4}{*}{$\begin{array}{c}\sigma_{3}^{\prime} \\
\mathrm{kPa}\end{array}$} & \\
\hline & & & & \\
\hline & & \multicolumn{3}{|c|}{$\mathrm{k} \operatorname{triax}(\mathrm{m} / \mathrm{s})$} \\
\hline & & $10 \mathrm{kPa}$ & $20 \mathrm{kPa}$ & 10 e $20 \mathrm{kPa}$ \\
\hline \multirow{2}{*}{ pre } & 160 & 7.9E-08 & $4.1 \mathrm{E}-08$ & $6.01675 \mathrm{E}-08$ \\
\hline & 360 & $4.2 \mathrm{E}-08$ & $3.5 \mathrm{E}-08$ & $3.83775 \mathrm{E}-08$ \\
\hline \multirow{2}{*}{ post } & 160 & $2.3 \mathrm{E}-09$ & $1.4 \mathrm{E}-09$ & $2.34914 \mathrm{E}-09$ \\
\hline & 360 & $1.8 \mathrm{E}-09$ & $8.2 \mathrm{E}-10$ & $1.32775 \mathrm{E}-09$ \\
\hline
\end{tabular}


Nel campo di sforzi investigato, la conducibilità resta pressoché invariata all'aumentare degli sforzi efficaci $\sigma_{3}^{\prime}$; i modesti decrementi sono pressoché paragonabili nei campioni naturali o trattati (Fig. 8).

Le conducibilità calcolate su campioni prossimi alla zona di iniezione sono significativamente inferiori a quelle dei terreni naturali (Fig. 8, Fig. 9). Dati i bassissimi valori in esame, se si esprime la riduzione della conducibilità idraulica attraverso l'espressione

$$
\Delta k=\frac{\log K_{p r e}-\log K_{p o s t}}{\log K_{p r e}}
$$

si quantifica una riduzione del $20 \%$.

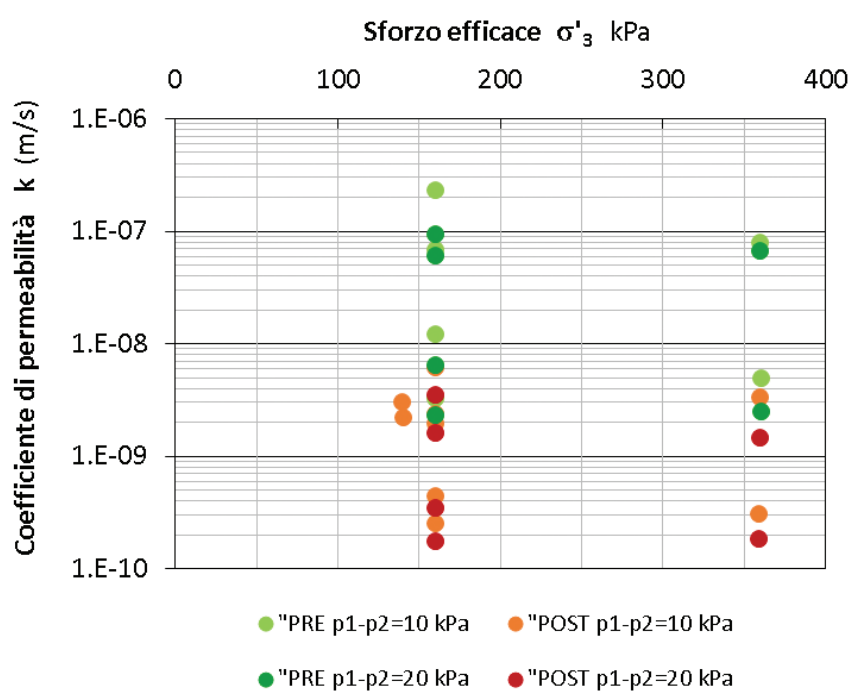

Fig. 8 - Coefficienti di permeabilità verticali, in relazione alla tensione verticale efficace $\sigma_{3}^{\prime}$, distinti sulla base della differenza di pressione dei pori imposta $(\Delta \mathrm{p}=10 \mathrm{kPa}$ o $\Delta \mathrm{p}=20 \mathrm{kPa})$. Sono plottati tutti i valori sia in carico che scarico. Confronto dei valori ottenuti su terreni naturali (simboli verdi) e su terreni trattati (simboli rossi e arancio).

Fig. 8 - Hydraulic vertical conductivity (by permeability tests in tiaxial cell) vs the vertical effective stress $\sigma_{3}^{\prime}$, distinguished on the basis of the imposed pore pressure difference ( $\Delta p=10 \mathrm{kPa}$ or $\Delta p=20 \mathrm{kPa})$. Values refer to both loading and unloading phases. Green symbols are related to natural soils while red and orange symbols to treated soils.

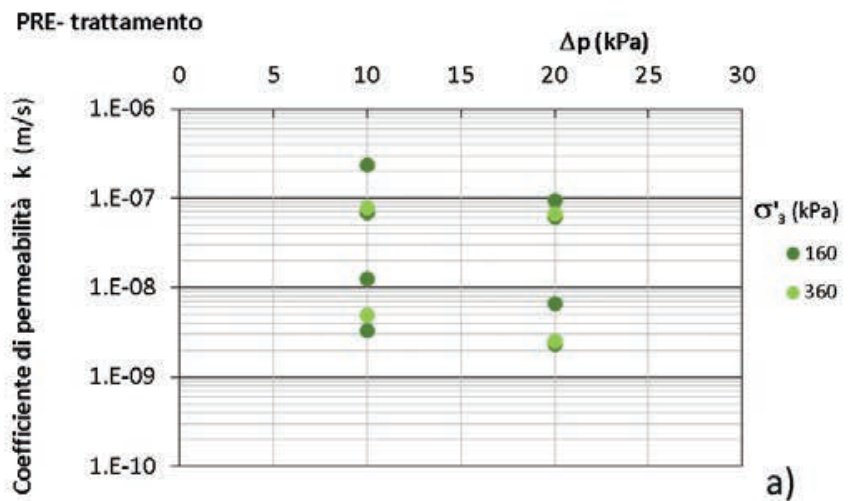

Anche i valori di $\mathrm{K}$ calcolati dalle curve di consolidazione confermano l'effetto del trattamento: valori più bassi di uno due ordini di grandezza sono calcolati per i terreni trattati (Tab.2). Già analizzando il comportamento deformativo registrato in fase di consolidazione si constatava che i terreni trattati raggiungono la fine consolidazione con minori variazioni di volume.

Il diagramma di Fig. 10 ci permette di riconoscere una riduzione delle permeabilità per effetto del trattamento che si manifesta anche senza una drastica variazione degli indici dei vuoti (e). La figura confronta infatti i valori di conducibilità nei campioni naturali e trattati in relazione all'indice dei vuoti determinato per ogni specifico gradino di carico alla fine della fase di consolidazione e per ogni prova di permeabilità.

Se consideriamo estremi (outlier) i valori a destra, riferiti a terreni trattati che mostrano bassa conducibilità idraulica anche per più alti valori di e, i restanti valori appaiono raggruppati in due cluster distinti in cui i punti riferiti ai terreni trattati sono caratterizzati da minor indice dei vuoti e minor conducibilità rispetto a quelli riferiti al terreno naturale. Inoltre si osserva che i più bassi valori di $\mathrm{k}$ nei terreni naturali sono riconducibili a valori più bassi di indice dei vuoti (relazione diretta), mentre nei trattati le variazioni di $\mathrm{k}$ risultano circoscritte in un campo più piccolo di variazione di e, senza una altrettanto chiara relazione diretta.

Il ristretto intervallo di variazione dell'indice dei vuoti e di conducibilità idraulica nei terreni naturali può attribuirsi alla modesta variabilità spaziale delle proprietà fisico-meccaniche riconoscibili già in condizioni naturali pre-trattamento; l'osservazione è coerente con la consistenza elevata del terreno in esame, con il suo elevato grado di sovraconsolidazione già in condizioni naturali pre-trattamento, nonché con il comportamento deformativo (modesta variazione di volume) registrato durante le fasi di consolidazione in cella triassiale.

Dalle osservazioni riportate è dunque ragionevole affermare che la riduzione di $\mathrm{k}$ indotta dal trattamento non è necessariamente prodotta dalla riduzione di e; basse $\mathrm{k}$ si misurano anche per e maggiori (vedi valori estremi di e nel grafico di Fig. 10).

\section{POST-trattamento}

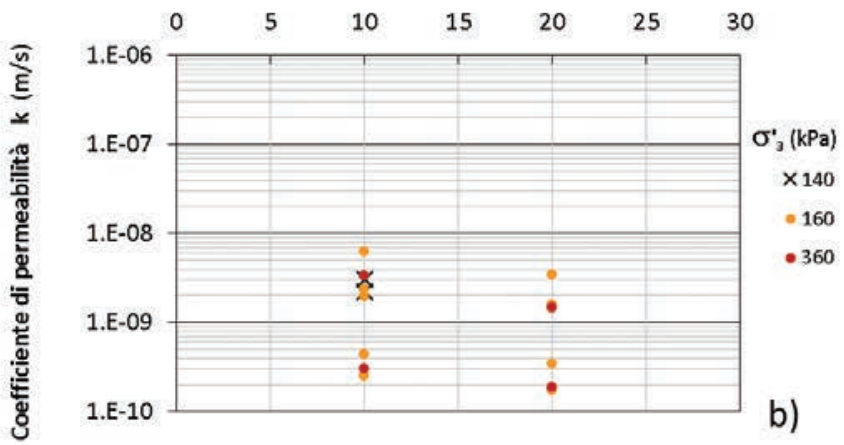

Fig. 9 - Coefficienti di permeabilità triassiale calcolati su terreni naturali (a) e su terreni trattati (b). Variazioni per differenza di pressione dei pori imposta $(\Delta \mathrm{p}=10 \mathrm{kPa}$ o $\Delta \mathrm{p}=20 \mathrm{kPa}$ ) e plottati in relazione alla tensione efficace $\sigma_{3}$. Sono plottati tutti i valori sia in carico che scarico.

Fig. 9 - Hydraulic conductivity (by permeability tests in three-axial cell) on natural soils (a) and treated soils (b). Variations with imposed pore pressure difference ( $\Delta p=10$ kPa 0 $\Delta p=20 k P a)$ and plotted in relation to the effective tension $\sigma_{3}^{\prime}$. Values refer to both loading and unloading phases. 
Il trattamento può produrre una compattazione con riduzione di volume che abbassa i valori di $\mathrm{k}$ o può anche impregnare il terreno riducendo la connettività dei vuoti e dunque la conducibilità idraulica. Allo stato attuale lo studio degli effetti del trattamento attraverso osservazioni mineralogicotessiturali (per esempio in microsonda elettronica a scansione SEM) non sono disponibili nella letteratura scientifica, ma sono oggetto di approfondimento del presente lavoro.

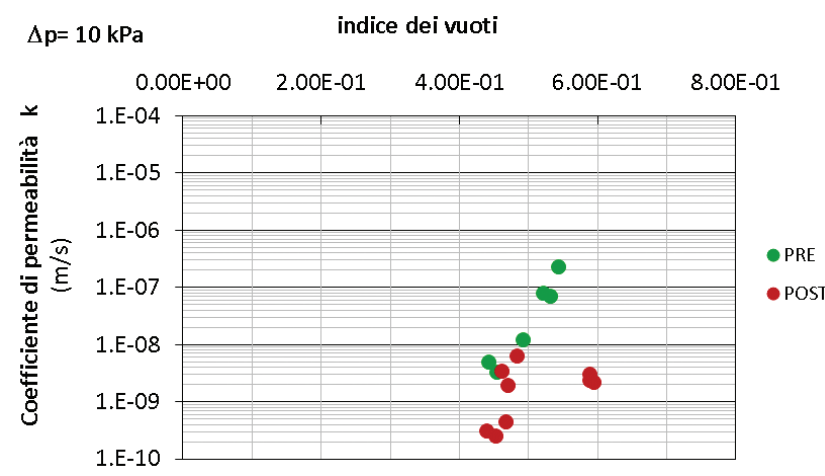

Fig. 10 - Coefficienti di permeabilità triassiale vs indice dei vuoti. Confronto dei valori ottenuti su terreni naturali (simboli verdi) e su terreni trattati (simboli rossi). Valori calcolati imponendo una differenza di pressione dei pori $\Delta \mathrm{p}=10 \mathrm{kPa}$. Sono plottati tutti i valori sia in carico che scarico.

Fig. 10 - Hydraulic conductivity (by permeability tests in three-axial cell) vs. voids ratio. Comparison of the values obtained on natural soils (green symbols) and on treated soils (red symbols). Values calculated by imposing a pore pressure difference $\Delta p=10 \mathrm{kPa}$. Values refer to both loading and unloading phases.

\section{Conclusioni}

La ricerca, svolta allo scopo di valutare gli effetti dei trattamenti di consolidamento con resine espandenti sulle proprietà idrauliche in terreni argillosi ha carattere sperimentale e, sebbene utilizzi strumenti di indagine comunemente in uso nei campi della geotecnica/idrogeologia, la loro applicazione alla problematica è del tutto originale.

Si sviluppa attraverso la realizzazione di un campo prova sperimentale e comprende essenzialmente prove geotecniche/ idrogeologiche in laboratorio.

Diversamente da ciò che accade nella pratica reale, il piano campagna è scarico ovvero senza carico da costruzione edificata, ma la possibilità di realizzazione degli scavi pre e post trattamento fino ad una profondità appena al di sopra del punto di iniezione con resine espone sezioni stratigrafiche che consentono di visualizzare la distribuzione delle resine iniettate e le caratteristiche di impregnazione del terreno coesivo in esame, nonché supportare le risultanze dalle indagini di tomografia di resistività elettrica.

La ricerca presenta una certa complessità anche in conseguenza alla natura del terreno investigato (limi con argilla, debolmente sabbiosi) che già in condizioni naturali ha caratteri di elevata consistenza e bassissima permeabilità. Ciononostante le prove di permeabilità su provini indisturbati naturali e trattati riescono a far apprezzare come il trattamento sia in grado di modificare le caratteristiche di conducibilità idraulica del terreno. Certamente $i$ dati sperimentali presentati si riferiscono alla scala del campione macroscopico e non si può escludere un effetto scala. Approfondimenti su questo aspetto sono necessari ma non possono prescindere dalla conoscenza delle proprietà relative al macrocampione e indubbiamente meglio si potranno comprendere avendo qui potuto ispezionare direttamente la distribuzione delle resine. Le resine iniettate seguano in parte piani di debolezza preesistenti ed in parte impregnano in modo capillare masse omogenee e continue di terreno, conferendogli non solo maggior resistenza meccanica, come è documentato in Apuani et al 2015, ma anche minor conducibilità idraulica. Le indagini di resistività elettrica consentono di apprezzare i volumi affetti dal trattamento, notoriamente dipendenti dalle modalità di iniezione (numero, pressioni e quantità di resina), e di attribuire le variazioni delle proprietà misurate a quel volume.

Alla scala in esame la conducibilità idraulica del terreno trattato si riduce significativamente e, in considerazione del valore estremamente basso che il terreno già possiede in condizioni naturali, può essere espressa come una variazione logaritmica del $20 \%$.

I risultati ottenuti, ed in particolare il miglioramento delle proprietà di conducibilità, invitano a ulteriori riflessioni circa la possibilità di nuovi impieghi della tecnica di iniezione con resine espandenti in campo ambientale, per esempio in contesti di terreni e/o acque contaminate da sversamenti locali e puntuali, in genere affrontati applicando tecniche più tradizionali di trattamento di inertizzazione (Apuani et al 2006) o di contenimento dei flussi.

Ringraziamenti: Un ringraziamento speciale al Prof. Ing Gian Paolo Giani; la nostra gratitudine per aver avviato gli autori alla problematica, per il costante supporto scientifico e la grande umanità. Gli autori ringraziano Marco Perfido, tecnico del Laboratorio di Geologia Applicata: geotecnica e geomeccanica del Dip. di Scienze della Terra dell'Università degli Studi di Milano.

Acknowledgment: A special thank goes to Professor Gian Paolo Giani; our gratitude for having introduced the authors to the subject, for his constant scientific support and great humanity. Acknowledgements to Marco Perfido, technician of the Engineering Geology Laboratory for Testing Material of the Department of Earth Science, University of Milan. 


\section{BIBLIOGRAFIA}

AA.VV. (2001). Poliuretano, speciale applicazioni, ANPE - Studioemmesrl. "Polyurethane, special applications, ANPE"

AGI (Associazione Geotecnica Italiana) (1997). Raccomandazioni sulla programmazione ed esecuzione delle indagini geotecniche. "Recommendations on planning and execution of geotechnical investigations" (in Italian)

Apuani, T., Beretta, G. P., Pellegrini, R. (2006). Linee Guida per l'inertizzazione in situ dei suoli contaminati. "Guidelines for in situ inertization of contaminated soils" Provincia di Milano: Milan, IT, Vol. 12. Available on line http://www.cittametropolitana.mi.it/export/ export_14032014/p_bonifiche_lineeguida12_inertizzazione.pdf (in Italian)

Apuani T., Giani G. P.,D’Attoli M., Fischanger F., Ranieri G., Santarato G. (2011). Valutazione dell'efficienza delle iniezioni di resine espandenti per il consolidamento dei terreni di fondazione mediante prove geotecniche e indagini di tomografia elettrica tridimensionale. "Evaluation of the efficiency of expanding resin injections for the consolidation of foundation soils by geotechnical tests and three-dimensional electrical tomography surveys". Atti del XXIV Convegno Nazionale di Geotecnica "Innovazione Tecnologica nell'ingegneria Geotecnica", Napoli 22-24 giugno 2011, Vol 2, pp 309-316 (in Italian)

Apuani T., Giani G. P., d'Attoli M., Fischanger F., Morelli G., Ranieri G., and Santarato G. (2015). Assessment of the Efficiency of Consolidation Treatment through Injections of Expanding Resins by Geotechnical Tests and 3D Electrical Resistivity Tomography. The Scientific World Journal - Hindawi Publishing Corporation, Vol. June 2015, Article ID 237930, 13 pages http://dx.doi. org $/ 10.1155 / 2015 / 237930$

ASTM D1556-07. Standard Test Method for Density and Unit Weight of Soil in Place by the Sand-Cone Method. Annual Book of ASTM Standards. Soil and Rock; Building Stones; Geotextiles. Vol. 04

ASTM D2167-08. Standard Test Method for Density and Unit Weight of Soil in Place by the Rubber Balloon Method. Annual Book of ASTM Standards. Soil and Rock; Building Stones; Geotextiles. Vol. 04

ASTM D2216-10 Standard Test Methods for Laboratory Determination of Water (Moisture) Content of Soil and Rock by Mass Annual Book of ASTM Standards. Soil and Rock; Building Stones; Geotextiles. Vol. 04

ASTM D2487-11 Standard Practice for Classification of Soils for Engineering Purposes (Unified Soil Classification System). Annual Book of ASTM Standards. Soil and Rock; Building Stones; Geotextiles. Vol. 04

ASTM D2488 Description and Identification of Soils (Visual-Manual Procedure). Annual Book of ASTM Standards. Soil and Rock; Building Stones; Geotextiles. Vol. 04

ASTM D2937-10. Standard Test Method for Density of Soil in Place by the Drive-Cylinder. Annual Book of ASTM Standards. Soil and Rock; Building Stones; Geotextiles. Vol. 04

BS1377-1 Methods of test for soils for civil engineering purposes - Part 1: General requirements and sample preparation

BS 1377-5 Methods of test for Soils for civil engineering purposes -Part 5: Compressibility, permeability and durability tests

BS 1377-6:1990 Methods of test for Soil for civil engineering purposes - Part 6: Consolidation and permeability tests in hydraulic cells and with pore pressure measurement.

D'Attoli M. (2016) STRADE \& AUTOSTRADE (2016). La riqualifica strutturale di parte della pavimentazione rigida del piazzale di sosta degli aeromobili mediante iniezione controllata di resine espandenti." The structural upgrading of part of the rigid flooring of the aircraft parking area by controlled injection of expanding resins." Rapporto Tecnico.

Dei Svaldi A., Favaretti M., Pasquetto A., and Vinco G. (2005). Analytical Modelling of the Soil Improvement by Injections of High Expansion Pressure Resin. Bulletin für angewandte Geologie, Vol. 10 (2), pp. $71-81$
Fischanger, F., Morelli, G., LaBrecque, D. and Occhi, M. (2007). Monitoring resin injections with 3d Electrical Resistivity Tomography (ERT) using surface and multi-borehole electrode arrays. Proceedings of Symposium on the Application of Geophysics to Environmental and Engineering, Problems (SAGEEP), Denver, Colorado (USA), April 1-5, 2007, 1226-1233.

Fischanger, F., Occhi, M. (2009). La tomografia elettrica 3D nei consolidamenti del terreno con resine espandenti, " $3 D$ electric tomography in soil consolidation with expanding resins" V Congresso Regionale dei Geologi Regione Sicilia, Palermo (Italy), 26 - 27 Giugno, 2009.

Fischanger F., Morelli G., Ranieri G., Santarato G., and Occhi M. (2013). 4D cross-borehole electrical resistivity tomography to control resin injection for ground stabilization: A case history in Venice (Italy). Near Surface Geophysics, Vol. 11, pp. 41-50

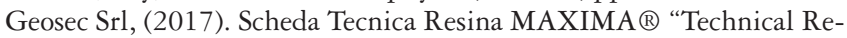
port Resin MAXIMA®"

ISPRA (Istituto Superiore per la Protezione e la Ricerca Ambientale) (2013). Carta Geologica d'Italia alla scala 1:50.000. Foglio 199, Parma SUD. Servizio Geologico d'Italia. "Geological Map of Italy scale 1:50.000. Table 199, South Parma" Italian Geological Survey. Available on line at http://www.isprambiente.gov.it/Media/ carg/199_PARMA_SUD/Foglio.html

Istituto Giordano s.p.a, (2017).Rapporto di prova N 340457 “Technical report $\mathrm{N}^{\circ} 340457$ "

Morelli G., LaBrecque D.J. (1996). Advances in ERT inverse modelling, European Journal of Environmental and Engineering Geophysical Society 1 (2), 171-186.

Morelli G., LaBrecque D.J. (1996). Advances in ERT inverse modelling, European Journal of Environmental and Engineering Geophysical Society 1 (2), 171-186.

Occhi, M., Occhi, A., Gualerzi, D. (2006). A method for homogenizing and stabilising a soil by way of injections Patent EP1914350.

Occhi, M., Occhi, A., Gualerzi, D.(2008). Local seismic protection method for existing and/or possible construction sites destined for the foundation areas and those surrounding the building construction. Patent EP1956147.

Occhi, M. (2014). Quaderno 4 - Resina Maxima ${ }^{\circledR}$ per il consolidamento dei terreni di fondazione "Maxima ${ }^{\circledR}$ resin for the consolidation of foundation soils" Technical Report.

Occhi, M., Occhi, A., Gualerzi, D.(2017). Method and kit for mitigating the risk of liquefaction of ground to be Consolidated - International Publication Number WO 2017/182939 Al

Oertel G., Hanser Publishers, Munich (1993). 770 pp., Polyurethane handbook (2nd ed.). DM 358, ISBN 3-446-17198-3

Rittgers, J.B., Sirles, P., Morelli, G. and Occhi, M., (2010). Case history: Monitoring resin injections with the aid of $4 \mathrm{D}$ geophysics. Proceedings of Symposium on the Application of Geophysics to Environmental and Engineering Problems (SAGEEP), Keystone, Colorado (USA), April 11-15, 2010, 379-390.

Santarato G., Ranieri G., Occhi M., Morelli G., Fischanger F., and Gualerzi D. (2011). Three-dimensional Electrical Resistivity Tomography to control the injection of expanding resins for the treatment and stabilization of foundation soils", Engineering Geology, Vol. 119, pp. 8-30

Santarato G., Albertini A., d'Attoli M., Navi F., Occhi M., Fischanger M., Morelli M., Leoni M., Apuani T., Loddo F. and Ranieri G. (2017). Soil Stabilization: Types, Methods and Applications Chapter 3. The Consolidation and Stabilization of Foundation Soils through the Injection of Expanding Polyurethane Resin under a Non-Invasive Diagnostic Check by 3d-4d-Ert (pp. 165-230) ISBN 978-1-53612-507-8

Vincent, M. (2009). Projet ARGIC: Analyse du Retrait-Gonflement et de ses Incidences sur les Constructions."Project ARGIC: Analysis of the Withdrawal-Swelling and its Impact on Constructions" Projet ANR05-PRGCU-005, Rapport Final, Rapport BRGM/RP-57011, (in French). 